\title{
Diagnostic potential of extracellular vesicle-associated microRNA-10b and tumor markers for lung adenocarcinoma
}

\author{
GUANGDA YUAN, HONGYA XIE, TENGTENG WEI, DONGLIN ZHU, \\ CHUANYU ZHANG and YONG YANG
}

Department of Thoracic Surgery, The Affiliated Suzhou Hospital of Nanjing Medical University, Suzhou, Jiangsu 215000, P.R. China

Received December 29, 2020; Accepted May 21, 2021

DOI: 10.3892/ol.2021.12875

\begin{abstract}
MicroRNAs (miRNAs/miRs) in extracellular vesicles (EVs) are potential diagnostic markers. The purpose of the present study was to investigate potential EV miRNA biomarkers for lung adenocarcinoma (LUAD). Potential miRNAs were identified by searching public databases and verified by examining clinical samples. The diagnostic value of EV-associated miR-10b, plasma miR-10b and tumor markers (TMs), including $\alpha$-fetoprotein (AFP), neuron-specific enolase, carcinoembryonic antigen (CEA), cytokeratin 19 fragment 21-1 (CYFRA211), pro-gastrin-releasing-peptide, carbohydrate antigen (CA)125, CA153, CA199 and CA724, was evaluated via receiver operating characteristic curve analysis. By searching the Gene Expression Omnibus and The Cancer Genome Atlas databases, miR-10b was identified as a potential biomarker. The analysis of clinical samples suggested that EV-associated miR-10b from plasma was significantly differentially expressed between LUAD and control samples. EV-associated miR-10b could function as a diagnostic marker for LUAD, with an AUC of 0.998, which was higher than the AUCs for TMs such as AFP, CEA, CYFRA211, CA125, CA153, CA199, CA724, pro-gastrin-releasing-peptide and neuron-specific enolase. In conclusion, EV-associated miR-10b may be a potential diagnostic biomarker for LUAD that is superior to plasma miR-10b and TMs.
\end{abstract}

\section{Introduction}

Lung cancer is the most common malignant tumor in the world and the cancer with the highest morbidity and lethality

Correspondence to: Dr Yong Yang, Department of Thoracic Surgery, The Affiliated Suzhou Hospital of Nanjing Medical University, 16 Baitaxi Road, Suzhou, Jiangsu 215000, P.R. China E-mail: 337914338@qq.com

Key words: lung adenocarcinoma, extracellular vesicle, microRNA, tumor marker rates, causing $>1$ million deaths every year (1). In addition, lung cancer causes earlier mortality than other cancer types, decreasing the patient lifespan by an average of 5.75 years (2). In China in 2015, lung cancer had morbidity and mortality rates of 57.26 per 100,000 and 45.87 per 100,000 individuals, respectively (3). According to histological classification, lung cancer has four major types: Adenocarcinoma, squamous cell carcinoma, large cell carcinoma and small cell carcinoma. Among all the subtypes of lung cancer, lung adenocarcinoma (LUAD) is the most heterogeneous and aggressive subtype (4). In the past few decades, the incidence of LUAD has increased and it has become one of the most common lung cancer types (5). LUAD accounts for $40 \%$ of all lung cancer cases. Symptomatic LUAD is usually diagnosed at an advanced stage, and $>80 \%$ of patients cannot be operated on. Screening and treatment of early stage LUAD can effectively decrease the mortality rate (6). However, the utilization of traditional chest X-rays, sputum tests and bronchoscopies is limited in lung cancer screening due to their low detection rate and high discomfort level. Low-dose computed tomography (LDCT) screening is a better choice for lung cancer screening. LDCT examination is a fast procedure and the radiation dose is significantly lower than that of conventional chest $\mathrm{CT}$, being equivalent to the effective radiation dose of chest X-rays. Moreover, the clarity of the chest structure and the sensitivity and accuracy of chest lesions detected by LDCT are greater than that of conventional chest X-rays. In China, the utilization of LDCT results in a significant increase in the detection of early-stage lung cancer, especially in females $(7,8)$. Unfortunately, the low number of healthcare providers that can perform LDCT and the high price of this test limit its widespread use (9).

Liquid biopsy includes the detection of tumor markers (TMs), circulating tumor DNA, circulating tumor cells and other molecules. This type of biopsy is non-invasive and has been widely used in recent years (10). Detection of TMs in the blood was first employed in 1963, when $\alpha$-fetoprotein (AFP) and carcinoembryonic antigen (CEA) were determined to be cancer markers (11). With the development of monoclonal antibody technology, hundreds of TMs have been discovered and widely used for detection clinically. AFP is a glycoprotein 
and is employed as a cancer biomarker, particularly in liver cancer (12). CEA is an oncofetal protein and a cell adhesion molecule that is associated with tumor invasion, dissemination, metastasis and immune suppression (13). The serum CEA level conveys prognostic information and can predict recurrence and patient mortality in lung cancer cases $(14,15)$. Carbohydrate antigen (CA)125, CA153, CA199 and CA724 are all membrane antigens that have been reported to be useful biomarkers in patients with lung, breast and ovarian cancer $(16,17)$. Cytokeratin 19 fragment 21-1 (CYFRA211) is a cell structural protein that can be released into the blood from degraded tumor cells (18). Elevated serum CYFRA211 levels have been found in a variety of organs, including the lungs and liver, and in the breast (19-21). The glycolytic enzyme neuron-specific enolase (NSE) mainly exists in the central and neuroendocrine tissues. NSE has been reported as a useful marker in both non-small cell lung cancer (NSCLC) and SCLC (22).

MicroRNAs (miRNAs/miRs) are small non-coding RNAs of $\sim 20$ nucleotides that regulate target gene expression at either the translational or post-transcriptional level and can also function as biomarkers (23). miRNAs are dysregulated in numerous types of diseases, particularly cancer. It has been reported by some researchers that circulating miRNAs in the plasma or serum could be potential biomarkers and therapeutic targets for LUAD. The miR-200 family could function as a prognostic marker of LUAD (24). miR-1205 may also be a predictor of overall survival (OS) in LUAD (25). In addition, miR-198 has been observed to serve as an independent prognostic factor for survival (26). However, the complex internal environment limits the application of miRNAs. For example, RNase is abundant in vivo, which could result in RNA degradation (27). Extracellular vesicles (EVs) are small vesicles that are shed from all types of live cells. EVs contain proteins, mRNAs, miRNAs and other molecules, and function to transport them to distant target cells. EV cargo varies depending on the health of the cell and the pathological state. The membrane structure of EVs provides protection for delivery of these molecules and ensures their stability, allowing these molecules to function as TMs (28). The present study searched for potential markers of LUAD via bioinformatics analysis and determined that miR-10b may be a good candidate. Next, the expression of miR-10b was determined in both plasma and EVs, and the diagnostic power of miR-10b was then compared with that of TMs such as AFP and CEA.

\section{Materials and methods}

Subjects and sample collection. Between May 2018 and June 2020, 80 subjects who were diagnosed with LUAD and 69 control subjects were recruited from The Affiliated Suzhou Hospital of Nanjing Medical University (Suzhou, China). The Ethics Review Board of The Affiliated Suzhou Hospital of Nanjing Medical University approved the study. Informed consent was obtained from all participants. The subjects were treated according to the principles described in the Declaration of Helsinki. Patient clinical parameters, including sex and age, were collected by reviewing the medical records.

For patients with LUAD, blood was drawn after diagnosis and before any treatment. Patients with a history of cancer or severe clinical symptoms and genetic diseases were excluded from the study. The control samples were obtained only from healthy subjects who came to the hospital for a physical examination and patients with benign lung disease, and not from patients with other tumor types. EDTA blood tubes were employed for blood collection. For plasma separation, blood samples were centrifuged at $1,600 \mathrm{x}$ g for $10 \mathrm{~min}$ at room temperature, and the plasma samples were stored in a refrigerator at $-80^{\circ} \mathrm{C}$ for later use.

EV isolation and identification. Before EV isolation, plasma samples were centrifuged at $2,000 \mathrm{x}$ g for $10 \mathrm{~min}$ at $4^{\circ} \mathrm{C}$ and $10,000 \times \mathrm{g}$ for $30 \mathrm{~min}$ at $4^{\circ} \mathrm{C}$ to separate out the cells and apoptotic bodies. Next, $400 \mu \mathrm{l}$ of $30 \%$ PEG4000 $(1 \mathrm{M} \mathrm{NaCl})$ was added and incubated with $800 \mu \mathrm{l}$ of supernatant for $3 \mathrm{~h}$ at $4^{\circ} \mathrm{C}$. After centrifugation at $3,000 \mathrm{x}$ g for $15 \mathrm{~min}$ at $4^{\circ} \mathrm{C}$, the pellet was subjected to further experiments. The Nano platform (IZON Science, Ltd.) was utilized to analyse the size distribution of the pellet.

Western blotting. Western blotting (WB) was employed to detect the signature proteins of EVs, including tumor susceptibility gene 101 protein (TSG101) and cluster of differentiation 9 (CD9), with GAPDH as the reference protein. Exosomes were lysed with RIPA buffer (Enzo Biochem, Inc.), and the protein concentrations were assayed using a BCA protein assay kit (Thermo Fisher Scientific, Inc.). Anti-TSG101 (catalog no. ab133586; 1:5,000), anti-CD9 (catalog no. ab223052; 1:5,000) and anti-GAPDH (catalog no. ab8245; 1:5,000) (all Abcam) were used as the primary antibodies. The detailed WB protocol was in keeping with a previously reported protocol (29). The density of the WB image was analyzed using ImageJ software (National Institutes of Health).

RNA isolation and $q P C R$. RNA isolation and $\mathrm{qPCR}$ were performed in accordance with the studies by Shan et al (30) and Su et al (31). RNAiso Plus (Takara Biotechnology Co., Ltd.) was employed to isolate RNA from EVs and plasma according to the manufacturer's instructions. In detail, $1 \mathrm{ml}$ RNAiso Plus was added to the pellets or $200 \mu 1$ plasma and mixed with a homogenizer. Next, $200 \mu \mathrm{l}$ chloroform was added and mixed with a vortex oscillator. The mixture was placed at room temperature for $10 \mathrm{~min}$ and centrifuged at $12,000 \mathrm{x} \mathrm{g}$ for $15 \mathrm{~min}$ at $4^{\circ} \mathrm{C}$. The supernatant was transferred to a new $1.5-\mathrm{ml}$ centrifuge tube and mixed with an equal volume of isopropyl alcohol. After overnight incubation at $-20^{\circ} \mathrm{C}$, the solution was centrifuged at $12,000 \mathrm{xg}$ for $15 \mathrm{~min}$ at $4^{\circ} \mathrm{C}$. The supernatant was discarded, and $75 \%$ ethanol was prepared with DEPC water to wash the RNA. After centrifugation at $12,000 \mathrm{xg}$ for $5 \mathrm{~min}$ at $4^{\circ} \mathrm{C}$, the remaining liquid was removed with blotting paper, and the inverted position was maintained on new blotting paper for 15-30 min. The RNA was dissolved in $10 \mu 1$ DEPC water.

A total of 5 control subjects and 5 patients with LUAD were selected to perform a preliminary experiment. A U6 snRNA Normalization RT-PCR Quantitation kit (Shanghai GenePharma Co., Ltd.) was employed to perform reverse transcription of the RNA. The RT-PCR system used a final reaction volume of $20 \mu \mathrm{l}$, which contained $4 \mu \mathrm{l}$ 5X MMLV RT Buffer, $0.75 \mu 1 \mathrm{dNTP}(10 \mathrm{mM}), 1.2 \mu 1$ miRNA RT primers $(1 \mu \mathrm{M})$, 
$0.25 \mu 1 \mathrm{RNasin}(40 \mathrm{U} / \mu \mathrm{l}), 0.2 \mu \mathrm{l}$ MMLV Reverse Transcriptase, $10 \mu \mathrm{l}$ RNA sample (1-3 $\mu \mathrm{g})$ and $3.6 \mu \mathrm{l}$ RNase-free $\mathrm{H}_{2} \mathrm{O}$. The standard thermocycling conditions were as follows: $25^{\circ} \mathrm{C}$ for $30 \mathrm{~min}, 42^{\circ} \mathrm{C}$ for $30 \mathrm{~min}, 85^{\circ} \mathrm{C}$ for $5 \mathrm{~min}$ and then storage at $4^{\circ} \mathrm{C}$. Next, $\mathrm{Cq}$ values were measured via $\mathrm{qPCR}$. Three Hairpin-it ${ }^{\mathrm{TM}}$ miRNAs qPCR Quantitation kit reagents boxes (Shanghai GenePharma Co., Ltd.) were employed to perform qPCR. All the primer sequences used in the study are listed in Table I. The qPCR system used a final reaction volume of $20 \mu$ l, which contained $10 \mu \mathrm{l} 2 \mathrm{X}$ Real-time PCR Master Mix Buffer (FAM), $0.4 \mu \mathrm{l}$ miRNA specific primer set $(10 \mu \mathrm{M})$, $0.4 \mu \mathrm{l}$ miRNA specific probe $(10 \mu \mathrm{M}), 0.4 \mu \mathrm{l}$ ROX reference dye (50X), $0.2 \mu \mathrm{l}$ Taq DNA polymerase (5 U/ $\mu \mathrm{l}), 2 \mu \mathrm{l}$ cDNA and $7.6 \mu \mathrm{l}$ sterilized $\mathrm{H}_{2} \mathrm{O}$. The standard thermocycling conditions were as follows: $95^{\circ} \mathrm{C}$ for $3 \mathrm{~min}$, followed by 40 cycles of $95^{\circ} \mathrm{C}$ for $12 \mathrm{sec}$ and $62^{\circ} \mathrm{C}$ for $40 \mathrm{sec}$. The signal was then collected at $62^{\circ} \mathrm{C}$. U6 snRNA was used as an internal reference for normalization, relative to which the expression of miRNAs was calculated using the comparison $2^{-\Delta \Delta \mathrm{Cq}}$ method (32). The EV-associated and plasma miR-10b, miR-200a and miR-141 expression levels in the LUAD and control groups were compared. Synthetic miR-10b was diluted to $10^{-3}, 10^{-4}, 10^{-5}$, $10^{-6}, 10^{-7}$ and $10^{-8} \mu \mathrm{M}$. RNA $(2 \mu \mathrm{l})$ from samples and different concentrations of synthetic miR-10b were reverse transcribed into cDNA and then were used to establish standard curve. The concentration of miR-10b in samples was calculated according to the established standard curve.

Detection of TMs. The AFP, NSE, CEA, CYFRA211, pro-gastrin-releasing-peptide (Pro-GRP), CA125, CA153, CA199 and CA724 were detected by the Department of Laboratory Medicine, The Affiliated Suzhou Hospital of Nanjing Medical University. Blood was drawn after diagnosis and before any treatment. After standing for $20 \mathrm{~min}$, the serum was separated by centrifugation at $1,600 \mathrm{x}$ g for $10 \mathrm{~min}$ at room temperature and electrochemiluminescence detection was used. The serum samples were tested on the same day. Results were obtained from the Department of Pathology.

Bioinformatics analysis. The Cancer Genome Atlas (TCGA) (https://www.cancer.gov/about-nci/organization/ccg/research/structural-genomics/tcga) survival analysis was performed with Kaplan-Meier Plotter (https://kmplot. com/analysis/) (33). The expression data of the target miRNA in the GSE114711 dataset (34) were obtained from the Gene Expression Omnibus (GEO) database (https://www.ncbi. nlm.nih.gov/geo/) using R v3.5.3 (https://cran.r-project. org/bin/windows/base/old/3.5.3/). Target gene prediction was performed using TarBase (35), TargetScan (36) and microT-CDS (37). The function of target genes was analyzed via Kyoto Encyclopedia of Genes and Genomes (KEGG; https://www.kegg.jp/kegg/kegg1.html), Gene Ontology (GO; https://david.ncifcrf.gov/) annotation and protein-protein interaction (https://string-db.org/) analyses. The expression of genes was analyzed using UALCAN (38). The networks of target genes were constructed with Cytoscape v3.6.0 (http://chianti. ucsd.edu/cytoscape-3.6.0/) and GeneMANIA (39).

Statistical analysis. Data are presented as the mean \pm standard deviation. Experiments were repeated in triplicate. Data were
Table I. Primer sequences used for quantitative PCR.

Gene Primer sequences (5'- 3')

$\begin{array}{ll}\text { U6 snRNA } & \text { Forward: CTCGCTTCGGCAGCACA } \\ & \text { Reverse: AACGCTTCACGAATTTGCGT }\end{array}$

miR-10b Forward: AACCCATACCCTGTAGAACCGAA Reverse: GTGCAGGGTCCGAGGT

miR-141 Forward: AGACCTCACCTGGCCTGTGGCC Reverse: GAACCCACCCGGGAGCCATCTT

miR-200a Forward: TAACACTGTCTGGTAACGATGT Reverse: CATCTTACCGGACAGTGCTGGA

miR, microRNA; snRNA, small nuclear RNA.

analyzed using SPSS v.19 (IBM Corp.). A non-parametric Mann-Whitney U test was employed to compare miRNA expression levels between the control and LUAD groups. A Wilcoxon signed-rank test was employed to compare miRNA expression levels between EVs and plasma. A non-parametric Kruskal-Wallis test was employed to compare miRNA expression levels at different stages. Bonferroni's correction was used for the correction of multiple comparisons. $\mathrm{P}<0.05$ was considered to indicate a statistically significant difference. Pearson's correlation analysis was employed to analyze the correlation between miRNA expression levels and age. A receiver operating characteristic (ROC) curve and the area under the ROC curve (AUC) were established to assess the potential of miR-10b to distinguish LUAD patients from individuals in the control group. Kaplan-Meier plotter (https://kmplot. com/analysis/) was used to analyze the survival curve of miRNAs (33). The OS from 0 to 120 months of patients with LUAD and control subjects was calculated from data recorded in the TCGA database. The distributions of OS time were analyzed using the Kaplan-Meier method and compared using a two-sided log-rank test.

\section{Results}

Description of subjects. In total, 149 patients were enrolled in this study, including 69 control subjects and 80 patients with LUAD (Table II). The age of the patients ranged from 21 to 82 years old. There were 66 female patients and 83 male patients. Of the 80 patients with LUAD, 64 had early stage disease (stage I + II). The number of patients with lesions located in the right or left lobe was similar. A total of 70 patients had only 1 lesion, 2 patients had 2 lesions and only 1 patient had 3 lesions.

EV isolation and identification. The procedure for isolating EVs is shown in Fig. 1A. The size distribution analysis indicated that the pellets contained vesicles ranging in size from 80 to $280 \mathrm{~nm}$ (Fig. 1B). Signature proteins, including TSG101 and CD9, and the reference GAPDH, were detected by WB analysis (Fig. 1C). The results of the density analysis are shown in Fig. S1. The WB results indicated that the pellets obtained by PEG precipitation did contain EVs, and there was 
Table II. Summary of patient characteristics $(n=149)$.

\begin{tabular}{|c|c|}
\hline Characteristic & Value \\
\hline \multicolumn{2}{|l|}{ Diagnosis, n (\%) } \\
\hline Control & $69(46.3)$ \\
\hline LUAD & $80(53.7)$ \\
\hline \multicolumn{2}{|l|}{ Age, years } \\
\hline Mean \pm SD & $59.93 \pm 13.26$ \\
\hline Range & $21-82$ \\
\hline \multicolumn{2}{|l|}{ Sex, n $(\%)$} \\
\hline Female & $66(44.3)$ \\
\hline Male & $83(55.7)$ \\
\hline \multicolumn{2}{|l|}{ Stage, n (\%) } \\
\hline I & $58(38.9)$ \\
\hline II & $16(10.7)$ \\
\hline III & $5(3.4)$ \\
\hline Unknown & $1 \quad(0.7)$ \\
\hline \multicolumn{2}{|l|}{ Lobe location, n (\%) } \\
\hline Left lower & $18(12.1)$ \\
\hline Left upper & $21(14.1)$ \\
\hline Right lower & $15(10.1)$ \\
\hline Right middle & $5(3.4)$ \\
\hline Right upper & $19(12.8)$ \\
\hline Right upper, lower & $1 \quad(0.7)$ \\
\hline Right upper, middle & $1 \quad(0.7)$ \\
\hline \multicolumn{2}{|l|}{ Lesions, n (\%) } \\
\hline 1 & $70(47.0)$ \\
\hline 2 & $9(6.0)$ \\
\hline 3 & $1 \quad(0.7)$ \\
\hline
\end{tabular}

LUAD, lung adenocarcinoma.

no significant difference in relative protein expression between the LUAD group and control group.

Search for potential miRNA markers. Obtained from the GEO database, the GSE114711 dataset contains small-RNA sequencing data for plasma-derived EVs from a cohort of patients with NSCLC at different stages (34). These patients included 7 control smokers, 11 patients with early stage NSCLC and 8 patients with late-stage NSCLC. Among the 3,762 detected miRNAs, there were no miRNAs that with significantly increased expression. However, 7 miRNAs in early stage disease and 33 miRNAs in late-stage disease exhibited significantly decreased expression compared with the control group, and 16 miRNAs showed significantly decreased expression in the late stages compared with the early stages. Using the expression in the control group as a reference, the expression of these miRNAs is shown in Fig. 2A. As the 80 outpatients with LUAD were primarily early stage lung cancer patients, a further comparison was made of the expression levels of the 7 miRNAs in the early stage and control groups (Table SI). The results showed that miR-10b, miR-141 and miR-200a were the 3 miRNAs that exhibited the most change in expression.
A

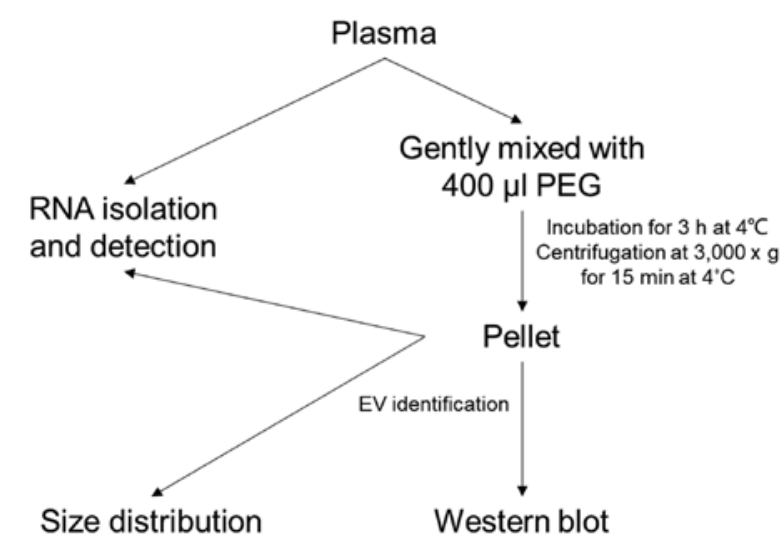

\section{B}

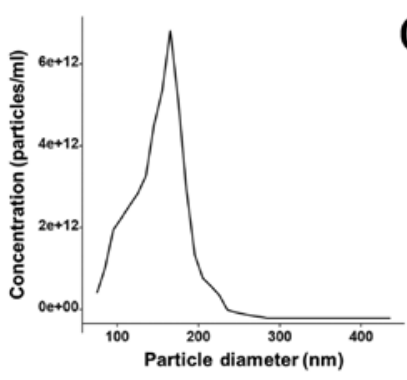

C

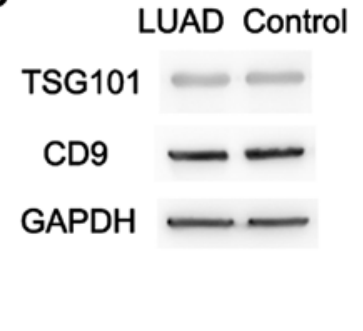

Figure 1. EV isolation and identification. (A) The procedure for EV isolation. (B) The size distribution of EVs. (C) Western blotting results for EVs. EV, extracellular vesicle; TSG101, tumor susceptibility gene 101 protein; CD9, cluster of differentiation 9; LUAD, lung adenocarcinoma.

The expression levels of miR-10b, miR-141 and miR-200a were then measured in 5 control subjects and 5 patients with LUAD (Fig. 2B-D). The non-parametric Mann-Whitney $\mathrm{U}$ test showed that the expression levels of plasma or EV miR-10b and miR-141 in the LUAD group were significantly higher than those in the control group $(\mathrm{P}<0.05)$, while there was no significant difference in miR-200a expression level. The Wilcoxon signed-rank test showed that there was no significant difference between the plasma and EV miR-10b and miR-141 expression levels in the control or LUAD groups $(\mathrm{P}>0.05)$. For miR-200a, there was no significant difference between plasma and $\mathrm{EV}$ in the control group, but there was a significant difference in the LUAD group $(\mathrm{P}=0.043)$. After Bonferroni's correction, there was no significant difference in miR-200a level in the LUAD group ( $P>0.05)$. Kaplan-Meier plotter was used to analyze the survival curve of miR-10b and miR-141 (Fig. 2E and F). miR-10b had a hazard ratio (HR) of $1.39(\mathrm{P}=0.039)$, while miR-141 had an HR of $1.32(\mathrm{P}=0.082)$. Finally, miR-10b was selected for further research.

Detection of miR-10b in plasma and EVs. Synthetic miR-10b was diluted for standard curve establishment (Fig. S2). The expression level of miR-10b in EVs and plasma was significantly increased in the LUAD group compared with that in the control group (Fig. 3A and B). The expression levels of miR-10b at different stages were also compared and the non-parametric Kruskal-Wallis test results showed that the expression levels of miR-10b did not exhibit a significant difference between stages I, II and III in EVs or plasma (Fig. 3C and D). The expression levels of miR-10b were also analyzed in patients with different sexes and ages. The results showed 

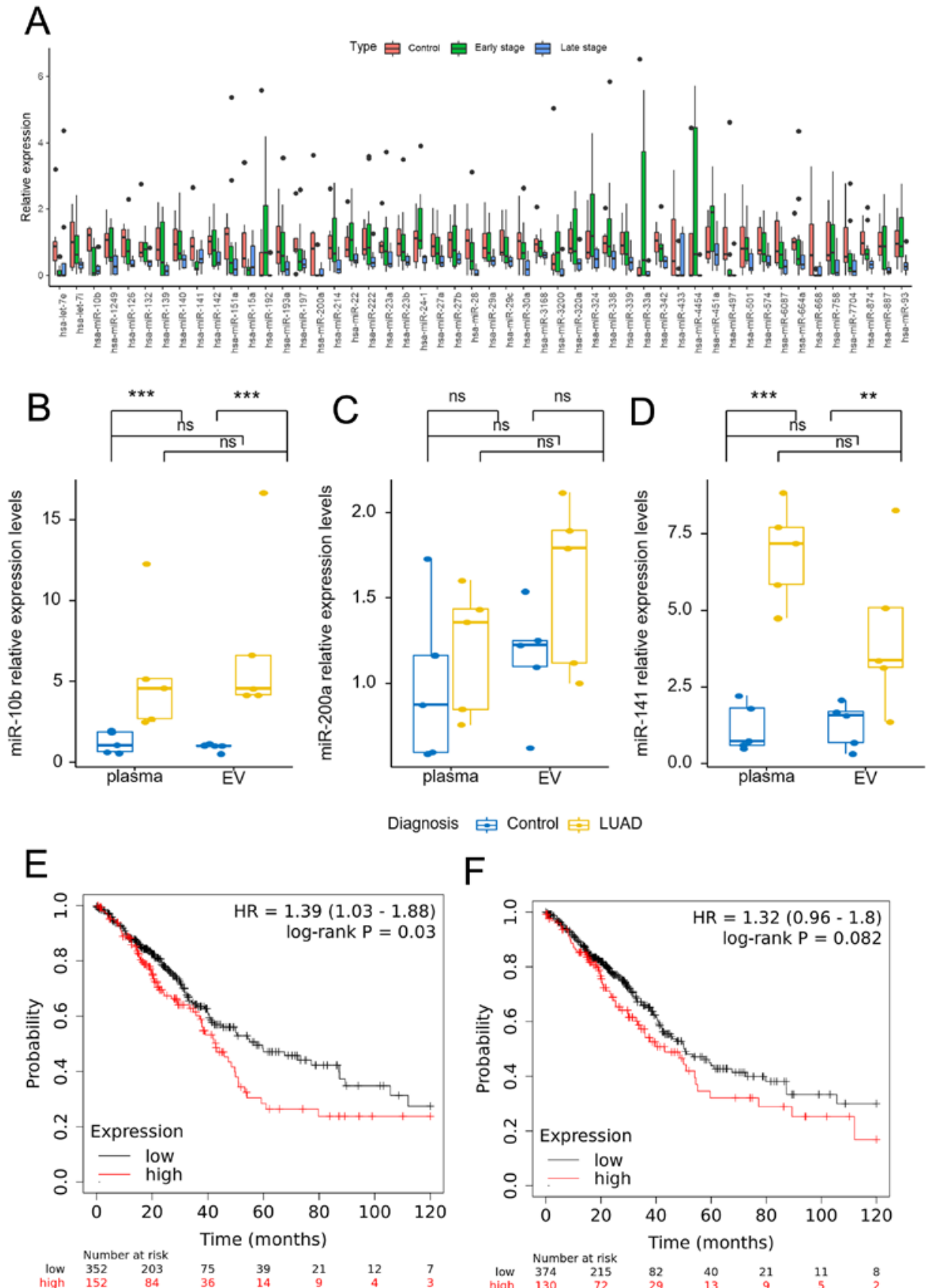

Figure 2. Search for potential miRNA markers. (A) The expression levels of miRNA in the control group, early stage LUAD group and late-stage LUAD group. (B) The expression levels of miR-10b in plasma and EVs from the control and LUAD groups. (C) The expression levels of miR-200a in plasma and EVs from the control and LUAD groups. (D) The expression levels of miR-141 in plasma and EVs from the control and LUAD groups. (E) The survival curve of miR-10b in LUAD. (F) The survival curve of miR-141 in LUAD. ${ }^{* *} \mathrm{P}<0.05$ and ${ }^{* * *} \mathrm{P}<0.001$. miRNA/miR, microRNA; LUAD, lung adenocarcinoma; EV, extracellular vesicle.

that the expression levels of miR-10b in EVs and plasma did not show a significant difference between males and females (Fig. S3A and B). The correlation between miR-10b and age was also analysed, and the results showed that there was no linear correlation. The correlation coefficients were 0.084 and 0.073 , respectively (Fig. S3C and D).

Detection of TMs. A total of 5 volunteers from the 69 patients of the control group and all LUAD subjects underwent the TM tests, which included AFP, NSE, CEA, CYFRA211, Pro-GRP, CA125, CA153, CA199 and CA724 assays (Fig. 4). The threshold of these markers is marked by dotted grey lines in the figures. Among these markers, CYFRA211 (22/80) and NSE (20/80) had the highest detection rates in the LUAD group (Fig. 4C and I). CA125, CA724 and NSE were determined to be abnormal in the control groups (Fig. 4D, G and I). The normal range of each marker is shown in Table III.

Comparison of miR-10b and TMs. ROC curve analysis was employed to compare the diagnostic efficiencies of miR-10b and TMs. miR-10b in EVs had a significantly higher AUC 
A

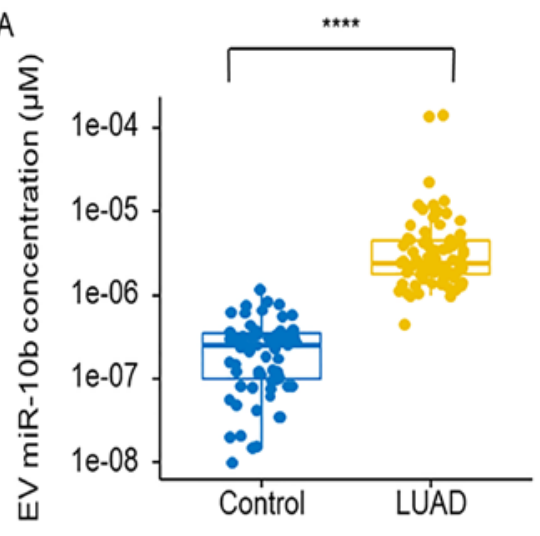

C

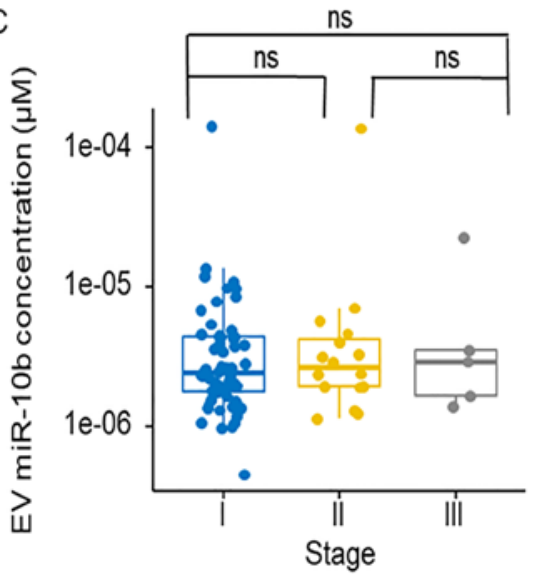

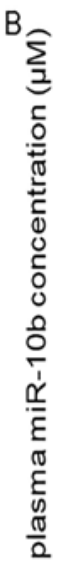

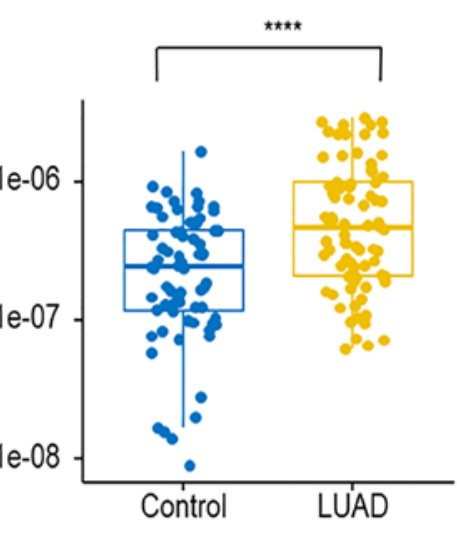

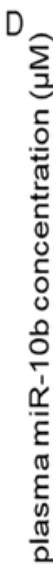

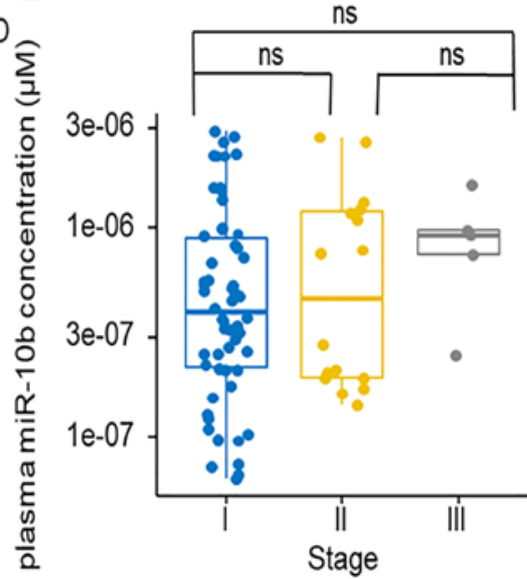

Figure 3. Expression levels of miR-10b in EVs and plasma. (A) The expression levels of miR-10b in EVs. (B) The expression levels of miR-10b in plasma. (C) The expression levels of EV miR-10b at different stages. (D) The expression levels of plasma miR-10b at different stages. ${ }^{* * * *} \mathrm{P}<0.0001$. miRNA/miR, microRNA; LUAD, lung adenocarcinoma; EV, extracellular vesicle.

$(\mathrm{AUC}=0.998)$ than that in plasma $(\mathrm{AUC}=0.693)(\mathrm{P}<0.01$; Fig. 5A). The Youden index was employed to determine the cut-off value. The sensitivity and specificity of EV-associated miR-10b (sensitivity, 98.75\%; specificity, 98.55\%) and plasma miR-10b (sensitivity, 37.5\%; specificity, 94.2\%) reached a maximum when the cut-off values were $9.087 \times 10^{-7}$ and $7.389 \times 10^{-7} \mu \mathrm{M}$, respectively. According to the cut-off value, the new AUCs of EV and plasma miR-10b were 0.986 and 0.658 , respectively (Fig. 5B). The ROC curves of TMs are shown in Fig. 5C. Among these markers, CA153 (AUC=0.844), CA199 $(\mathrm{AUC}=0.7275)$ and GRP $(\mathrm{AUC}=0.655)$ had the top 3 AUCs. After threshold modification, CYFRA211 had the highest AUC of 0.635 (Fig. 5D).

Bioinformatics analysis of miRNA and target genes. The target genes of miR-10b were predicted using TargetScan, microT-CDS and TarBase. There were 14 interaction target genes (Fig. 6A). These genes formed a network that included coexpression, colocalization and genetic interactions (Fig. 6B). Next, KEGG pathway enrichment analysis was performed and it was found that the 'circadian rhythm' pathway and the 'longevity regulating pathway' were the most enriched pathways (Fig. 6C). GO analysis was also performed. 'Gland development', 'thymus development' and 'positive regulation of protein complex assembly' were the top 3 biological processes (Fig. 6D). The 'RNA polymerase II transcription factor complex' and the 'nuclear transcription factor complex'
Table III. Normal reference ranges of TMs.

\begin{tabular}{lc}
\hline TM & Range \\
\hline AFP & $0-10 \mathrm{ng} / \mathrm{ml}$ \\
NSE & $0-20 \mathrm{ng} / \mathrm{ml}$ \\
CEA & $0-5 \mathrm{ng} / \mathrm{ml}$ \\
CYFRA211 & $0-3.5 \mathrm{ng} / \mathrm{ml}$ \\
Pro-GRP & $0-75 \mathrm{U} / \mathrm{ml}$ \\
CA125 & $0-35 \mathrm{U} / \mathrm{ml}$ \\
CA153 & $0-30 \mathrm{U} / \mathrm{ml}$ \\
CA199 & $0-34 \mathrm{U} / \mathrm{ml}$ \\
CA724 & $0-8.2 \mathrm{U} / \mathrm{ml}$ \\
\hline
\end{tabular}

AFP, $\alpha$-fetoprotein; NSE, neuron-specific enolase; CEA, carcinoembryonic antigen; CYFRA211, cytokeratin 19 fragment 21-1; Pro-GRP, pro-gastrin-releasing-peptide; CA, carbohydrate antigen; TM, tumor marker.

were the top 2 cellular components (Fig. 6E). 'Transcriptional activator activity, RNA polymerase II transcription regulatory region sequence-specific DNA binding' and 'transcription coactivator binding' were the most enriched molecular functions (Fig. 6F). 
A

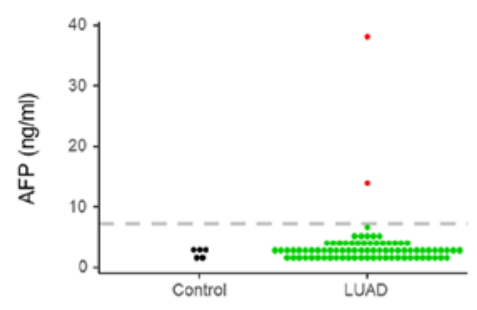

D

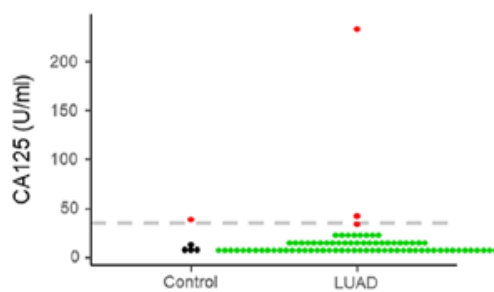

G

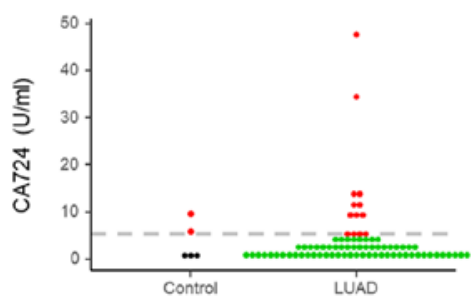

B

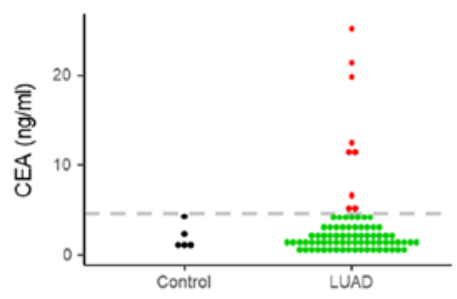

E

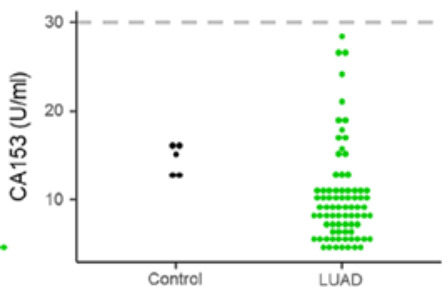

H

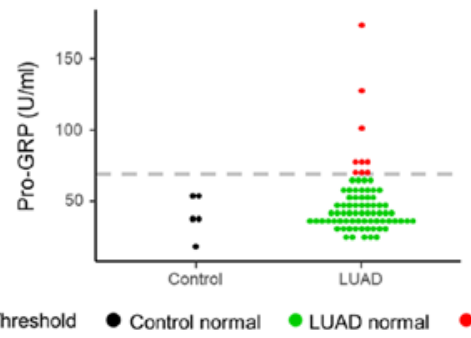

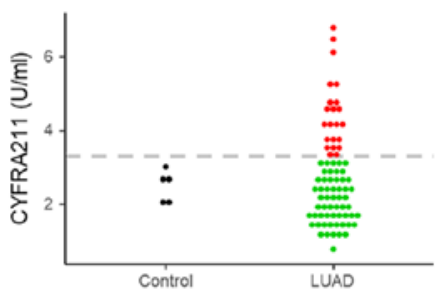

F

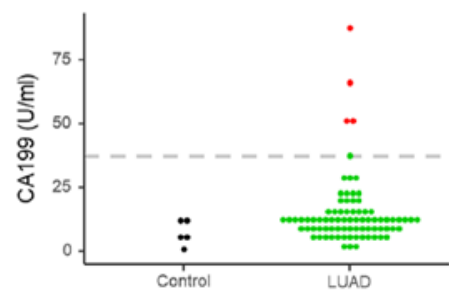

I

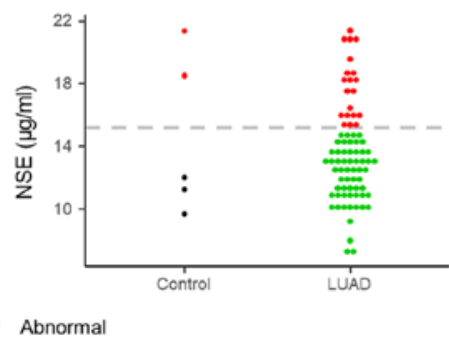

Figure 4. Levels of tumor markers. The levels of (A) AFP, (B) NSE, (C) CEA, (D) CYFRA211, (E) Pro-GRP, (F) CA125, (G) CA153, (H) CA199 and (I) CA724. According to common thresholds, a red dot indicates abnormal results, a black dot indicates normal results in the control group, and a green dot indicates normal results in the LUAD group. AFP, $\alpha$-fetoprotein; NSE, neuron-specific enolase; CYFRA211, cytokeratin 19 fragment 21-1; Pro-GRP, pro-gastrin-releasing-peptide; $\mathrm{CA}$, carbohydrate antigen; LUAD, lung adenocarcinoma.
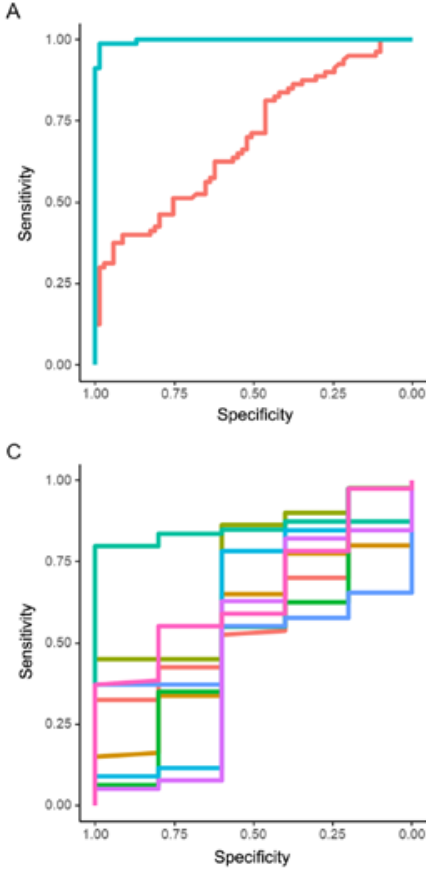

B
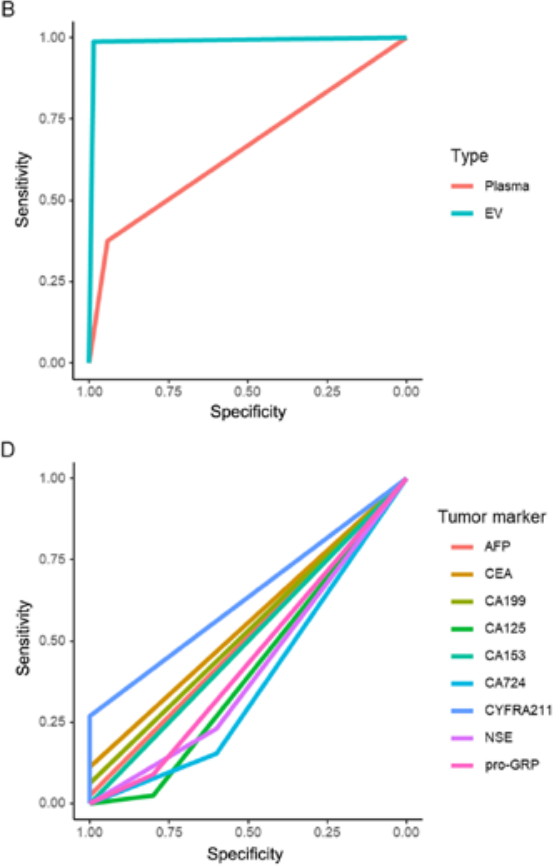

Figure 5. ROC curve analysis of miR-10b in EVs and plasma, and analysis of TMs. (A) ROC curve of miR-10b in EVs and plasma. (B) ROC curve of miR-10b in EVs and plasma adjusted by thresholds. (C) ROC curve of TMs. (D) ROC curve of TMs adjusted by thresholds. TM, tumor marker; AFP, $\alpha$-fetoprotein; NSE, neuron-specific enolase; CYFRA211, cytokeratin 19 fragment 21-1; Pro-GRP, pro-gastrin-releasing-peptide; CA, carbohydrate antigen; EV, extracellular vesicle; miR, microRNA; ROC, receiver operating characteristic. 


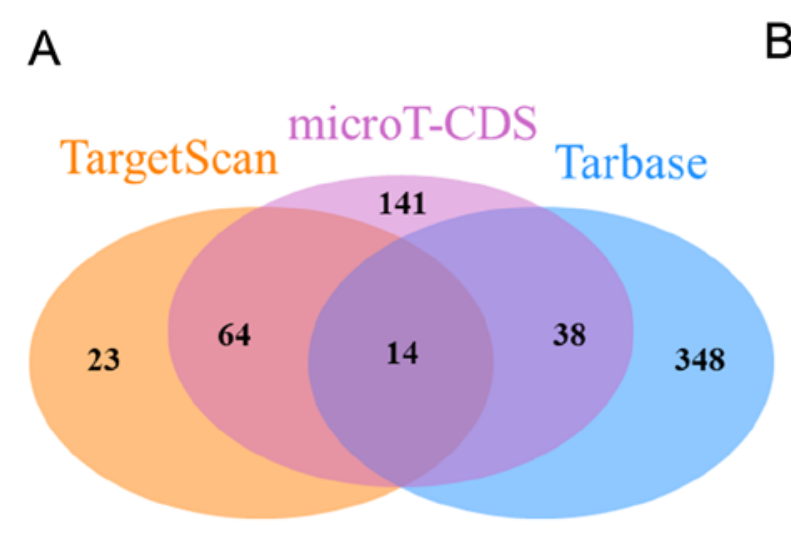

B

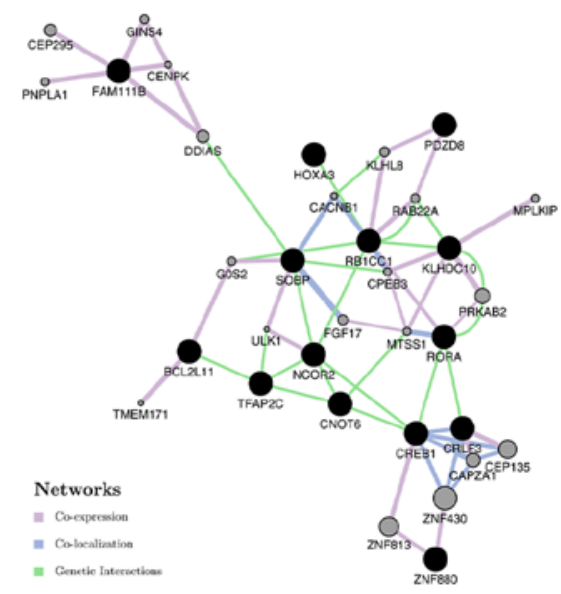

C

D

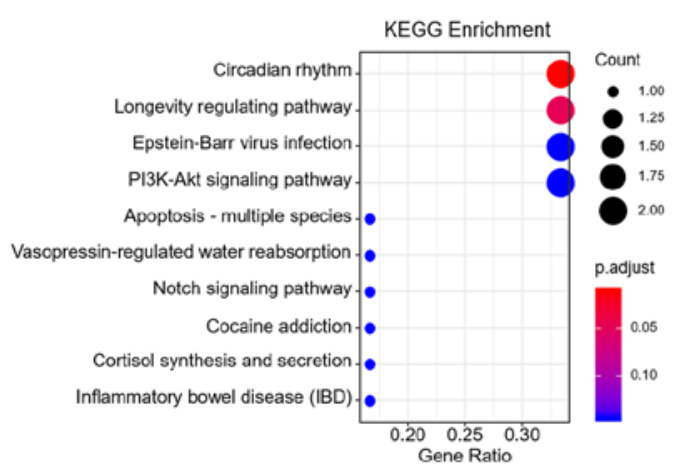

E

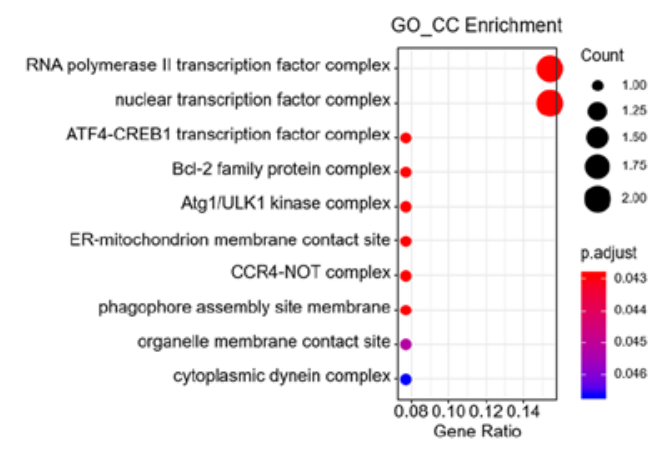

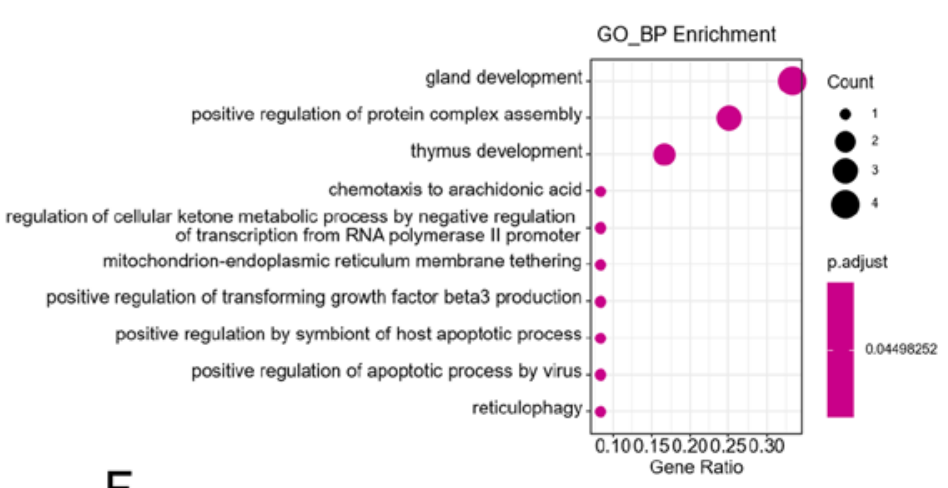

$\mathrm{F}$

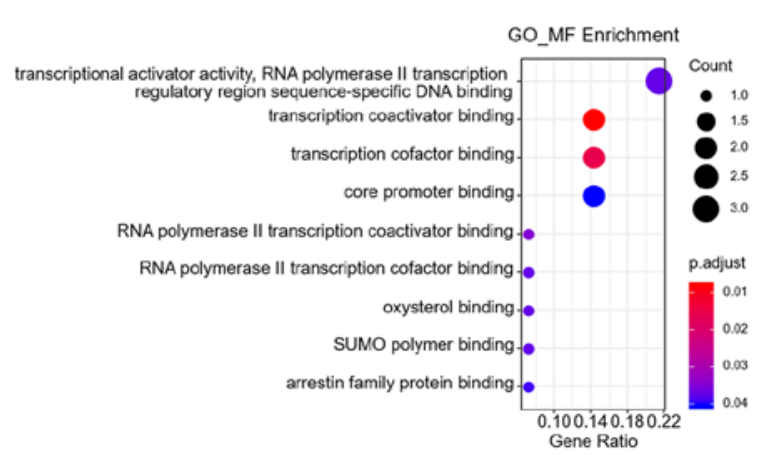

Figure 6. Bioinformatics analysis of miR-10b and its target genes. (A) Prediction of miR-10b target genes and their connections. (C) KEGG pathway analysis of target genes. (D) GO analysis of the biological processes of target genes. (E) GO analysis of the cellular components of target genes. (F) GO analysis of the molecular functions of target genes. KEGG, Kyoto Encylopedia of Genes and Genomes; GO, Gene Ontology; miR, microRNA; BP, biological process; $\mathrm{CC}$, cellular component; MF, molecular function.

\section{Discussion}

In China, the incidence of lung cancer continues to increase rapidly, which imposes a severe social and economic burden (40). In the present study, public databases were used to search for target miRNAs and miR-10b was determined to be a possible useful candidate for LUAD. TCGA database was also employed to verify the relationship between miR-10b and survival. The results showed that high miR-10b expression was associated with shorter survival time in LUAD. Next, clinical blood samples were used for further verification, and miR-10b was upregulated in LUAD samples in both plasma and EVs. The expression levels of miR-10b in plasma and EVs were not related to sex or age. The detailed reasoning as to why miR-10b expression is higher in LUAD is still unclear and requires further research.

In addition to traditional imaging examinations, including $\mathrm{X}$-ray and LDCT, other molecular tests have also been applied in LUAD detection. CYFRA211, CEA and NSE have been recorded as upregulated in the peripheral blood from patients with LUAD and may be used as diagnostic and prognostic markers for the disease $(14,41)$. In the present study, 22 CYFRA211, 20 NSE and 9 CEA abnormalities were found in the patients with LUAD. Moreover, in the control group, 2 samples showed abnormal NSE levels, and the highest abnormal levels in the control and LUAD groups were similar (20.79 and $21.41 \mu \mathrm{g} / \mathrm{ml}$, respectively). Subjects with a high abnormal NSE level in the control group need long-term monitoring to prevent the disease (42). 
ROC curve analysis was employed to compare the diagnostic value of miR-10b in plasma or EVs and TMs. miR-10b in both plasma and EVs showed significant differences between the control and LUAD groups. ROC curve analysis showed that EV-associated miR-10b was superior to plasma. Among the nine TMs, CA153 had the highest AUC of 0.844. However, the AUC was decreased to 0.5 when CA153 was adjusted by the common cut-off value of $30 \mathrm{U} / \mathrm{ml}$. CA153 is a recommended marker for breast cancer (17). The present data indicated that CA153 may be a marker for LUAD, but it requires an appropriate cut-off value. Even with the best cut-off, CA153 was still inferior to EV-associated miR-10b.

Bioinformatics analysis of miR-10b and its target genes was also performed to determine their potential functions. There were 14 target genes of miR-10b included in all the three databases. 'Circadian rhythm' was found to be the key KEGG pathway. It has been reported that circadian rhythm disruption can promote lung tumorigenesis (43). GO BP, CC and MF analyses showed that RNA polymerase II might have key involvement. RNA polymerase II participates in the transcription of mRNA and a number of non-coding RNAs in eukaryotic genomes, which is necessary for almost all life activities (44). These regulatory networks indicate new directions for further research.

The sample size is a limitation of this study. A total of 80 subjects who were diagnosed with LUAD and 69 control subjects were enrolled, and it took $>2$ years to collect these eligible patients. In the future, the sample size will be increased to ensure more accurate results. Besides the sample size, the detailed reasoning as to why miR-10b expression is higher in LUAD is still unclear. Huang et al (45) found that silencing of miR-10b inhibited tumor cell progress by arresting cell cycle progression in the $\mathrm{G}_{0} / \mathrm{G}_{1}$ phase and promoted apoptosis in NSCLC cells. EV-associated miR-10b in LUAD may also be involved in this pathway.

In conclusion, EV-associated miR-10b may be a potential biomarker for LUAD diagnosis and may be superior to plasma miR-10b and TMs. In addition, with an appropriate threshold, CA153 may also serve as a diagnostic biomarker for LUAD.

\section{Acknowledgements}

Not applicable.

\section{Funding}

No funding was received.

\section{Availability of data and materials}

All data generated or analyzed during this study are included in this published article.

\section{Authors' contributions}

GY and HX collected the patient data and performed the bioinformatics analysis. TW, DZ and CZ performed the experiments. YY and GY contributed to the study design and manuscript writing. All authors have read and approved the manuscript. GY and YY confirm the authenticity of all the raw data. All authors read and approved the final manuscript.

\section{Ethics approval and consent to participate}

Blood sample and clinical data collection were approved by the Ethics Review Board of The Affiliated Suzhou Hospital of Nanjing Medical University (Suzhou, China). Oral informed consent for publication was obtained from all participants, as approved by the Ethics Review Board.

\section{Patient consent for publication}

Not applicable.

\section{Competing interests}

The authors declare that they have no competing interests.

\section{References}

1. Siegel R, Ward E, Brawley O and Jemal A: Cancer statistics, 2011: The impact of eliminating socioeconomic and racial disparities on premature cancer deaths. CA Cancer J Clin 61: 212-236, 2011.

2. Yan Y, Chen Y, Jia H, Liu J, Ding Y, Wang H, Hu Y, Ma J, Zhang $X$ and Li S: Patterns of Life Lost to Cancers with High Risk of Death in China. Int J Environ Res Public Health 16: 2175-2193, 2019.

3. Zheng RS, Sun KX, Zhang SW, Zeng HM, Zou XN, Chen R, Gu XY, Wei WW and He J: Report of cancer epidemiology in China, 2015. Zhonghua Zhong Liu Za Zhi 41: 19-28, 2019 (In Chinese).

4. Zhang H, Guo L and Chen J: Rationale for Lung Adenocarcinoma Prevention and Drug Development Based on Molecular Biology During Carcinogenesis. OncoTargets Ther 13: 3085-3091, 2020.

5. Zhang X, Wu L, Xu Y, Zhang B, Wu X, Wang Y and Pang Z: Trends in the incidence rate of lung cancer by histological type and gender in Sichuan, China, 1995-2015: A single-center retrospective study. Thorac Cancer 9: 532-541, 2018.

6. Dama E, Melocchi V, Mazzarelli F, Colangelo T, Cuttano R, Di Candia L, Ferretti GM, Taurchini M, Graziano P and Bianchi F: Non-Coding RNAs as Prognostic Biomarkers: A miRNA Signature Specific for Aggressive Early-Stage Lung Adenocarcinomas. Noncoding RNA 6: 48, 2020.

7. Yang W, Qian F, Teng J, Wang H, Manegold C, Pilz LR, Voigt W, Zhang Y, Ye J, Chen Q, et al; Written on behalf of the AME Thoracic Surgery Collaborative Group: Community-based lung cancer screening with low-dose CT in China: Results of the baseline screening. Lung Cancer 117: 20-26, 2018.

8. Liang F, Wu C, Gu H, Zhu M, Xuan Z, Jiang Y, Chen H, Fu C and Zheng Y: Lung cancer incidence in female rises significantly in urban sprawl of Shanghai after introduction of LDCT screening. Lung Cancer 132: 114-118, 2019.

9. He D, Yu H and Chen Y: Equity in the distribution of CT and MRI in China: A panel analysis. Int J Equity Health 12: 39-53, 2013.

10. Siravegna G, Mussolin B, Venesio T, Marsoni S, Seoane J, Dive C, Papadopoulos N, Kopetz S, Corcoran RB, Siu LL, et al: How liquid biopsies can change clinical practice in oncology. Ann Oncol 30: 1580-1590, 2019.

11. Sell S: Cancer markers of the 1990 s. Comparison of the new generation of markers defined by monoclonal antibodies and oncogene probes to prototypic markers. Clin Lab Med 10: 1-37, 1990.

12. He Y, Lu H and Zhang L: Serum AFP levels in patients suffering from 47 different types of cancers and noncancer diseases. Prog Mol Biol Transl Sci 162: 199-212, 2019.

13. Beauchemin $\mathrm{N}$ and Arabzadeh A: Carcinoembryonic antigen-related cell adhesion molecules (CEACAMs) in cancer progression and metastasis. Cancer Metastasis Rev 32: 643-671, 2013.

14. Grunnet M and Sorensen JB: Carcinoembryonic antigen (CEA) as tumor marker in lung cancer. Lung Cancer 76: 138-143, 2012. 
15. Lei L, Chen Q, Wang Z, Han N, Chen B, Qin J and Lu HY: Usefulness of carcinoembryonic antigen in the diagnosis of smal cell lung cancer combined with adenocarcinoma. Adv Clin Exp Med 26: 1091-1094, 2017.

16. Nakamura $\mathrm{H}$ and Nishimura T: History, molecular features, and clinical importance of conventional serum biomarkers in lung cancer. Surg Today 47: 1037-1059, 2017.

17. Li X, Xu Y and Zhang L: Serum CA153 as biomarker for cancer and noncancer diseases. Prog Mol Biol Transl Sci 162: 265-276, 2019.

18. Buccheri G and Ferrigno D: Lung tumor markers of cytokeratin origin: An overview. Lung Cancer 34 (Suppl 2): S65-S69, 2001.

19. Uenishi T, Kubo S, Hirohashi K, Tanaka H, Shuto T, Yamamoto T and Nishiguchi S: Cytokeratin-19 fragments in serum (CYFRA 21-1) as a marker in primary liver cancer. Br J Cancer 88: 1894-1899, 2003.

20. Narita T, Funahashi H, Imai T, Takagi H and Kannagi R: Cytosol and serum concentration of cytokeratin subunit-19 fragment (cyfra-21-1) in breast-cancer. Oncol Rep 1: 747-750, 1994.

21. Kammer MN, Kussrow AK, Webster RL, Chen H, Hoeksema M, Christenson R, Massion PP and Bornhop DJ: Compensated Interferometry Measures of CYFRA 21-1 Improve Diagnosis of Lung Cancer. ACS Comb Sci 21: 465-472, 2019.

22. Yang G, Xiao Z, Tang C, Deng Y, Huang H and He Z: Recent advances in biosensor for detection of lung cancer biomarkers. Biosens Bioelectron 141: 111416, 2019.

23. Gu Y, Liu S, Zhang X, Chen G, Liang H, Yu M, Liao Z, Zhou Y, Zhang CY, Wang T, et al: Oncogenic miR-19a and miR-19b co-regulate tumor suppressor MTUS1 to promote cell proliferation and migration in lung cancer. Protein Cell 8: 455-466, 2017.

24. Yuan L, Bing Z, Yan P, Li R, Wang C, Sun X, Yang J, Shi X, Zhang $\mathrm{Y}$ and Yang $\mathrm{K}$ : Integrative data mining and meta-analysis to investigate the prognostic role of microRNA-200 family in various human malignant neoplasms: A consideration on heterogeneity. Gene 716: 144025-144025, 2019.

25. Dai B, Kong D-L, Tian J, Liu T-W, Zhou H and Wang ZF: microRNA-1205 promotes cell growth by targeting APC2 in lung adenocarcinoma. Eur Rev Med Pharmacol Sci 23: 1125-1133, 2019.

26. Wang SS, Fang YY, Huang JC, Liang YY, Guo YN, Pan LJ and Chen G: Clinical value of microRNA-198-5p downregulation in lung adenocarcinoma and its potential pathways. Oncol Lett 18: 2939-2954, 2019.

27. Sheu-Gruttadauria J, Pawlica P, Klum SM, Wang S, Yario TA, Schirle Oakdale NT, Steitz JA and MacRae IJ: Structural Basis for Target-Directed MicroRNA Degradation. Mol Cell 75: 1243-1255.e7, 2019

28. Barile L and Vassalli G: Exosomes: Therapy delivery tools and biomarkers of diseases. Pharmacol Ther 174: 63-78, 2017.

29. Kowal EJK, Ter-Ovanesyan D, Regev A and Church GM: Extracellular Vesicle Isolation and Analysis by Western Blotting. Methods Mol Biol 1660: 143-152, 2017.

30. Shan B, Ai Z, Zeng S, Song Y, Song J, Zeng Q, Liao Z, Wang T, Huang $\mathrm{C}$ and $\mathrm{Su}$ D: Gut microbiome-derived lactate promotes to anxiety-like behaviors through GPR81 receptor-mediated lipid metabolism pathway. Psychoneuroendocrinology 117: 104699, 2020.

31. Su D, Liao Z, Feng B, Wang T, Shan B, Zeng Q, Song J and Song Y: Pulsatilla chinensis saponins cause liver injury through interfering ceramide/sphingomyelin balance that promotes lipid metabolism dysregulation and apoptosis. Phytomedicine 76: 153265, 2020.
32. Livak KJ and Schmittgen TD: Analysis of relative gene expression data using real-time quantitative PCR and the 2(-Delta Delta C(T)). Methods 25: 402-408, 2001.

33. Nagy Á, Lánczky A, Menyhárt O and Győrffy B: Validation of miRNA prognostic power in hepatocellular carcinoma using expression data of independent datasets. Sci Rep 8: 9227-9235, 2018.

34. Nigita G, Distefano R, Veneziano D, Romano G, Rahman M, Wang K, Pass H, Croce CM, Acunzo M and Nana-Sinkam P: Tissue and exosomal miRNA editing in Non-Small Cell Lung Cancer. Sci Rep 8: 10222-10229, 2018.

35. Karagkouni D, Paraskevopoulou MD, Chatzopoulos S, Vlachos IS, Tastsoglou S, Kanellos I, Papadimitriou D, Kavakiotis I, Maniou S, Skoufos G, et al: DIANA-TarBase v8: A decade-long collection of experimentally supported miRNA-gene interactions. Nucleic Acids Res 46D: D239-D245, 2018

36. Agarwal V, Bell GW, Nam JW and Bartel DP: Predicting effective microRNA target sites in mammalian mRNAs. eLife 4: e05005-e05042, 2015

37. Paraskevopoulou MD, Georgakilas G, Kostoulas N, Vlachos IS, Vergoulis T, Reczko M, Filippidis C, Dalamagas $\mathrm{T}$ and Hatzigeorgiou AG: DIANA-microT web server v5.0: Service integration into miRNA functional analysis workflows. Nucleic Acids Res 41W: W169-W173, 2013

38. Chandrashekar DS, Bashel B, Balasubramanya SAH, Creighton CJ, Ponce-Rodriguez I, Chakravarthi BVSK and Varambally S: UALCAN: A Portal for Facilitating Tumor Subgroup Gene Expression and Survival Analyses. Neoplasia 19: 649-658, 2017.

39. Warde-Farley D, Donaldson SL, Comes O, Zuberi K, Badrawi R, Chao P, Franz M, Grouios C, Kazi F, Lopes CT, et al: The GeneMANIA prediction server: Biological network integration for gene prioritization and predicting gene function. Nucleic Acids Res 38 (Suppl 2): W214-W220, 2010.

40. Hong QY, Wu GM, Qian GS, Hu CP, Zhou JY, Chen LA, Li WM, Li SY, Wang K, Wang Q, et al; Lung Cancer Group of Chinese Thoracic Society; Chinese Alliance Against Lung Cancer: Prevention and management of lung cancer in China. Cancer 121 (Suppl 17): 3080-3088, 2015.

41. Jiang $\mathrm{ZF}$, Wang $\mathrm{M}$ and $\mathrm{Xu}$ JL: Thymidine kinase 1 combined with CEA, CYFRA21-1 and NSE improved its diagnostic value for lung cancer. Life Sci 194: 1-6, 2018.

42. Isgrò MA, Bottoni $P$ and Scatena R: Neuron-Specific Enolase as a Biomarker: Biochemical and Clinical Aspects. Adv Exp Med Biol 867: 125-143, 2015

43. Papagiannakopoulos T, Bauer MR, Davidson SM, Heimann M, Subbaraj L, Bhutkar A, Bartlebaugh J, Vander Heiden MG and Jacks T: Circadian Rhythm Disruption Promotes Lung Tumorigenesis. Cell Metab 24: 324-331, 2016.

44. Schier AC and Taatjes DJ: Structure and mechanism of the RNA polymerase II transcription machinery. Genes Dev 34: 465-488, 2020.

45. Huang J, Sun C, Wang S, He Q and Li D: MicroRNA miR-10b inhibition reduces cell proliferation and promotes apoptosis in non-small cell lung cancer (NSCLC) cells. Mol Biosyst 11: 2051-2059, 2015

This work is licensed under a Creative Commons Attribution-NonCommercial-NoDerivatives 4.0 International (CC BY-NC-ND 4.0) License. 